\title{
Additive effect of mesenchymal stem cells and defibrotide in an arterial rat thrombosis model
}

\author{
Dilek Dilli, M.D., Assoc. Prof. ${ }^{a}$, Emine Kılıç, Ph.D. ${ }^{b, c}$, Nihat Yumuşak, Ph.D..$^{d}$, \\ Serdar Beken, M.D., Assoc. Prof. ${ }^{a}$, Duygu Uçkan Çetinkaya, M.D., Prof., , \\ Ramazan Karabulut, M.D., Assoc. Prof.e and Ayşegül Zenciroğlu, M.D., Assoc. Prof. ${ }^{a}$
}

\section{ABSTRACT}

Background/aim. In this study, we aimed to investigate the additive effect of mesenchymal stem cells (MSC) and defibrotide (DFT) in a rat model of femoral arterial thrombosis.

Methods. Thirty Sprague Dawley rats were included. An arterial thrombosis model by ferric chloride $(\mathrm{FeCl} 3)$ was developed in the left femoral artery. The rats were equally assigned to 5 groups: Group 1-Sham-operated (without arterial injury); Group 2-Phosphate buffered saline (PBS) injected; Group 3-MSC; Group 4-DFT; Group 5-MSC + DFT. All had two intraperitoneal injections of $0.5 \mathrm{ml}$ : the $1^{\text {st }}$ injection was $4 \mathrm{~h}$ after the procedure and the $2^{\text {nd }}$ one 48 $\mathrm{h}$ after the $1^{\text {st }}$ injection. The rats were sacrificed 7 days after the $2^{\text {nd }}$ injection.

Results. Although the use of human bone marrow-derived (hBM) hBM-MSC or DFT alone enabled partial resolution of the thrombus, combining them resulted in near-complete resolution. Neovascularization was two-fold better in hBM-MSC + DFT treated rats $(11.6 \pm 2.4$ channels) compared with the hBM-MSC $(3.8 \pm 2.7$ channels) and DFT groups (5.5 \pm 1.8 channels) $(P<0.0001$ and $P=0.002$, respectively). Conclusion. The combined use of hBM-MSC and DFT in a rat model of arterial thrombosis showed additive effect resulting in near-complete resolution of the thrombus.

Key words: Thrombosis, mesenchymal stem cell, defibrotide, rats.

http: / / dx.doi.org/10.5546/ aap.2017.eng.249

To cite: Dilli D, Kılıç E, Yumuşak N, et al. Additive effect of mesenchymal stem cells and defibrotide in an arterial rat thrombosis model. Arch Argent Pediatr 2017;115(3):249-256.

\section{E-mail address:}

Dilek Dilli, M.D.:

dilekdilli2@yahoo.com

Funding:

This project was

supported by The

Scientific And

Technological

Research Council Of

Turkey(TUBITAK),

ARDEB/SBAG, Project

No: 3001/114S237-2014.

Conflict of interest:

None.

Received: 9-23-2016

Accepted: 11-29-2016

\section{Abbreviations:}

- Anti-vascular endothelial growth factor receptor-1: anti-VEGFR-1.

- Anti-von Willebrand factor: antiVWF.

- Congenital heart disease: CHD.

- Defibrotide: DFT.

- Ferric chloride: FeCl3.

- Hematoxylin and eosin: H\&E.

- Human bone marrow-derived: hBM.

- Mesenchymal stem cells: MSC. phosphate buffered saline (PBS).

- Prostaglandin I2: PGI2.

- Thromboxane A2: TXA2.

\section{INTRODUCTION}

Femoral artery catheterization either in form of indwelling arterial catheters for continuous blood pressure monitoring and blood sampling or cardiac catheter for the diagnosis and treatment of children with congenital heart disease (CHD) represents frequent procedures in neonatal intensive care units. Catheter insertion may lead to local endothelial injury and cessation of blood flow in the femoral artery, creating an environment contributive to thrombosis. ${ }^{1-3}$ Arterial thrombosis may cause serious acute complications such as skin necrosis, and threatened limb or organ viability. Management of thrombosis ranges from the use of thrombolytics and anticoagulants to surgical intervention. Natural thrombus resolution occurs by a process of organization that is similar to that found in wound healing. ${ }^{4}$ It is thought that recanalization of the artery proceeds by the coalescence of these vascular channels. Influencing these processes may enable to enhanced resolution of the thrombus. ${ }^{5,6}$

Therapeutic benefits of mesenchymal stem cells (MSCs) at recanalization and neovascularization processes have been shown. ${ }^{7,9}$ MSCs can differentiate into distinctive end-stage cell types, such as those that fabricate specific mesenchymalderived tissues including bone, cartilage, muscle, bone marrow stroma, tendon/ligament, fat, dermis and other connective tissues. , $^{70,11}$ Because of these unique properties, MSCs could be a future option to treat various diseases. Studies on the potential use of stem cells in pediatric 
diseases have recently aroused interest among clinicians. ${ }^{12,13}$ MSCs have been shown to be able to differentiate into mature vascular endothelial cells and to participate in a variety of vascular reconstruction processes in both experimental studies and clinical treatments of limb ischemia, as well as in the construction of engineered vascular tissue.,

Defibrotide (DFT), a polydeoxyribonucleotide, has been found to modulate endothelial cell function and to have an antithrombotic effect on arteries, veins and capillaries. ${ }^{14}$ DFT increases prostaglandin I2 (PGI2) and PGE2 generation, while decreasing thromboxane A2 (TXA2) generation. ${ }^{15}$ Furthermore, DFT directly interferes with migration and tube formation of endothelial cells which is dependent on mTORp70S6k activity. ${ }^{16,17}$ Besides, DFT has different activities [pro-fibrinolytic, anti-thrombotic, thrombolytic, anti-ischemic, anti-shock and anti-atherosclerotic] that fit antiendothelial cell activation activity. ${ }^{14,18-20}$ Thus, our present study was designed to investigate the additive effect of human bone marrow-derived (hBM) MSC and DFT in an experimental rat model of arterial thrombosis. We hypothesized that this protocol would increase the frequency of circulating endothelial precursors and promote the formation of new blood vessels (neovascularization), thereby enhancing the resolution of thrombi, and building a novel treatment regimen for arterial thrombosis.

\section{MATERIALS AND METHODS \\ Animals}

Experiments were carried out on 30 male adult Sprague-Dawley rats, with weights ranging from 250 to $350 \mathrm{~g}$, and ages 8-9 weeks at the time of the procedure. The animals were allowed free access to water and standard laboratory rat chow. All procedures were approved by animal ethics committee of Ankara Training and Research Hospital Ethical Board (Ankara, Turkey).

Thrombosis model-Ferric chloride ( $\mathrm{FeCl} 3$ ) induced thrombosis

Rats were anesthetized with ketamine/ xylazine. Body temperature was monitored with a rectal probe and maintained at approximately $37^{\circ} \mathrm{C}$ with a heating pad throughout the experiment. Left femoral artery was exposed by oblique groin incision, separated from the adherent tissue. After a stabilization period, thrombosis was induced by topically applying a piece $(2 \times 1 \mathrm{~mm})$ of Whatman paper, soaked in $\mathrm{FeCl} 320 \%$ to the exposed rat femoral artery as previously described. ${ }^{21} \mathrm{~A}$ small piece of aluminum foil was kept below the artery prior to the topical application of $\mathrm{FeCl} 3$ so that it should not come in contact with the adjacent tissue or femoral nerve. The animals were left anesthetized for 30 minutes to allow thrombus to form. Then, the rats were recovered and allowed free access to water and normal chow.

\section{Study design}

A total of 30 rats were equally assigned to one of five study groups. The four groups carried the artery injury: 1) Sham (without arterial injury): administered $0.5 \mathrm{ml}$ phosphate buffered saline (PBS), 2) PBS: administered $0.5 \mathrm{ml}$ PBS, 3) hBM-MSC: administered $1 \times 10^{6} \mathrm{hBM}-\mathrm{MSC}$ suspended in $0.5 \mathrm{ml}$ PBS, 4) DFT: administered DFT (0.5 ml, $40 \mathrm{mg} / \mathrm{kg}), 5)$ hBM-MSC + DFT: administered simultaneously $1 \times 10^{6} \mathrm{hBM}-\mathrm{MSCs}$ suspended in $0.5 \mathrm{ml}$ PBS and DFT $(0.5 \mathrm{ml}, 40 \mathrm{mg} /$ $\mathrm{kg})$. Except for sham controls, the rats were subjected to thrombosis procedure. All the rats received two intraperitoneal injections with the corresponding solution: the $1^{\text {st }}$ injection was 4 $\mathrm{h}$ after the procedure and $2^{\text {nd }}$ one $48 \mathrm{~h}$ after the $1^{\text {st }}$ injection.

\section{Isolation and culture of hBM-MSCs}

Hacettepe University Institutional Review Board (Ankara, Turkey) approved isolation, characterization and labeling of hBM-MSCs from healthy BM transplant donors, for use in in-vivo models. The healthy children whom we extracted bone barrow were already transplant donors for their siblings. A total of $200-400 \mathrm{ml}$ (based on the weight of the recipient) of bone marrow were extracted for the recipient under general anesthesia. We only used $2 \mathrm{ml}$ of these bone marrow samples for the research. If a child was cooperative, informed consent was taken both the child and his/her parent.

hBM-MSCs were isolated and grown in culture at the PEDI-STEM Stem Cell laboratory of Hacettepe University using marrow samples obtained from a healthy, 12-y-old bone marrow transplant donor. An informed consent was obtained from the parents and $2 \mathrm{ml}$ of bone marrow was used. Differentiation assays and immunophenotypic profiling were performed for characterization of hBM-MSCs [22, 23] (Figure $1 A-B)$.

Labeling of hBM-MSCs with iron oxide particles. hBM-MSCs were allowed to grow until $80 \%-$ $90 \%$ confluence, before changing medium. 
Cells were magnetically labeled with ferrum oxide (Endorem; Guerbet, Villepinte, France) and complexed to poly-L-lysine (Sigma-Aldrich Chemical Co., St. Louis, MO), as previously described. ${ }^{24}$ (Figure 1C).

Immunophenotype of control hBM-MSCs and iron-labeled $h B M-M S C s$. Culture expanded control and labeled $(50 \mu \mathrm{g} / \mathrm{mL}$ iron and $0.375 \mathrm{~g} /$ $\mathrm{mL}$ poly-L-lysine for $48 \mathrm{~h}$ ) hBM-MSCs were immunophenotypically characterized by flow cytometry (Becton Dickinson FACS Aria; BD Biosciences, Sparks, MD). ${ }^{22}$

\section{Tissue harvesting}

Rats were sacrificed 7 days after the $2^{\text {nd }}$ injection, after anesthetized with ketamine/ xylazine. The left groin was opened and femoral artery was dissected. The artery specimens were harvested for a histopathological and immunohistochemical evaluation. Injured vessel samples from hBM-MSC + DFT group, and vessel, heart, liver, and lung samples from hBM-MSC group were obtained to visualize iron-labeled hBM-MSCs that homed in different organs.

\section{Histolopathologic evaluation}

Measurement of neovascularization

The femoral artery specimens that contained the thrombus were fixed in $4 \%$ paraformaldehyde overnight and embedded in paraffin wax. Transverse sections ( $5 \mu \mathrm{m}$ thick) were cut at $150 \mu \mathrm{m}$ intervals throughout the length of the sample and stained with hematoxylin and eosin (H\&E). Sections of tissues were evaluated at 20x, 40x, 60x and 100x magnifications and were digitized using a microscope-mounted camera
(Olympus DP 73 camera, Olympus BX53-DIC microscope; Tokyo, Japan).

Recanalization channels were identified as spaces, lined by flat endothelial-like cells, found among resolving thrombus and the artery wall and within the main body of the thrombus. Neovascularization was measured by counting the number of vascular channels within and around the thrombus (ie., channels that contain erythrocytes) in five fields to obtain the average number of vascular channels per viewed field (per 100x).

All the images were analyzed blindly. Neovascularization was compared among the treated animals in groups 3, 4 and 5.

\section{Immunohistochemical analyses:}

Five sections from each rat were cut on interval of $150 \mu$ and processed for each antibody for immunostaining. For immunohistochemical analyses, adherent cells were collected and incubated with the following antibodies: antivon Willebrand factor (anti-VWF) [1:200; 36B11, Leica Biosystems, Newcastle, UK], anti-Vascular endothelial growth factor receptor-1 [antiVEGFR-1, 1:100; ab32152, Abcam, Cambridge, UK], anti- $\mathrm{CD}_{68}$ [1:100; 514H12, Leica Biosystems, Newcastle, UK], anti-CD ${ }_{34}[1: 100 ;$ QBEnd / 10, Leica Biosystems, Newcastle, UK), and anti- $\mathrm{CD}_{133}$ (1:100; 18470-1-AP, Prointech Group, Rosemont, IL 60018, USA]. Anti-VWF and anti-VEGFR-1 were used to define endothelial cells migrated around the injured vessel wall and thrombus, and anti- $\mathrm{CD}_{68^{\prime}}$ anti- $\mathrm{CD}_{34^{\prime}}$ and anti- $\mathrm{CD}_{133}$ were used to show progenitor cell migration to injured area for new capillary formation.

Figure 1. (A) Differentiation of hBM-MSCs into the adipogenic lineage, stained with Oil Red O. (B) Differentiation of hBMMSCs into the osteogenic lineage, stained with Alizarin Red S. (C) Labeling of hBM-MSCs with iron oxide particles (50 $\mu \mathrm{g} /$ ml endorem Prussian. Phase-contrast micrographs in A-C (500x)

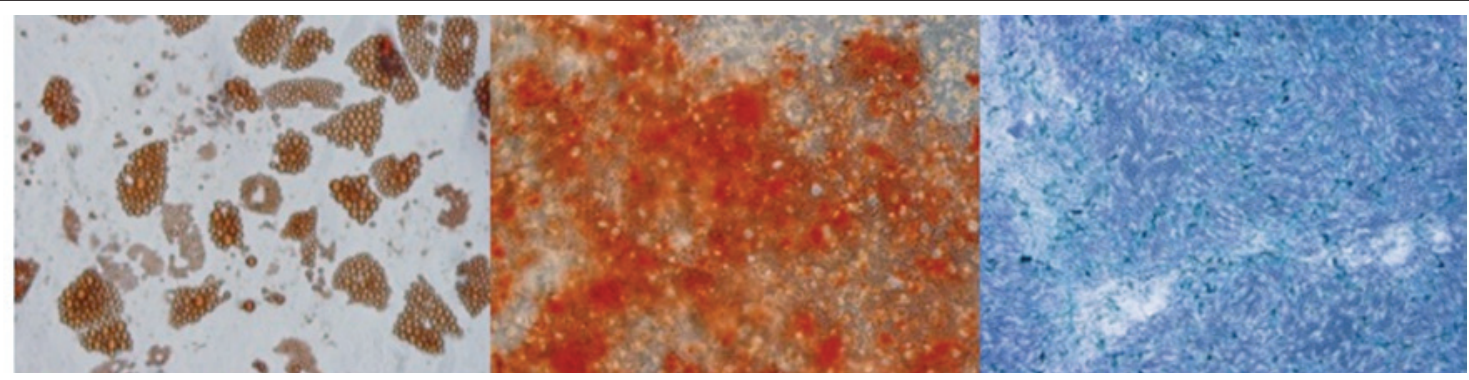

A) Adipogenic

B) Osteogenic

C) Iron labeled hBM-MSCs 
The number of antibody ${ }^{+}$cells per field in each section was counted in five fields at 100x magnification for VWF, CD34, CD68 and CD133 and at 40x for VEGFR-1. All counts were performed in a blind manner and average numbers of antibody+ cells per view field were obtained (Olympus DP 73 camera, Olympus BX53-DIC microscope; Tokyo, Japan).

Detection of iron-labeled hBM-MSCs in the tissues. Iron-labeled hBM-MSCs were detected with Prussian Blue staining to distinguish injected human cells from the host cells. After all procedures were completed, the stained sections were examined under a light microscope. Ferric reactive iron in the hBM-MSCs was visualized as blue.

Immunohistochemical examination of $h B M$ MSCs in tissues. Sections of $5 \mu \mathrm{m}$ thickness were processed with polylysine microscope slides. For the immunohistochemical examination, endogenous peroxidase activity was blocked with $3 \%$ hydrogen peroxide. Epitopes were stabilized with a serum blocking solution (Invitrogen, Carlsbad, CA). Sections were incubated with 3-2 microglobulin [B2M-02, ab27588, Abcam, Cambridge, UK] to detect cells of human origin. The slides were then counterstained with Mayer's hematoxylin and examined under a microscopemounted camera (Olympus DP 73 camera, Olympus BX53-DIC microscope; Tokyo, Japan). $\beta-2$ microglobulin positive hBM-MSCs were surrounded by brown-stained cell membranes.

\section{Statistical analysis.}

The SPSS statistical package (v15.0; SPSS, Inc., Chicago, IL) was used for the statistical analysis.
The mean \pm SD were used to display descriptive statistics. Group comparisons of independent variables were performed by independent $t$ test for two groups and one way ANOVA for three groups. Categorical variables were tested by $\kappa 2-$ test. Friedman's test was applied for intergroup comparisons of dependent variables. A $p<0.05$ was considered statistically significant. A power analysis was conducted; if 0.60 of the effect size and an alpha of 0.05 were used to obtain $80 \%$ power, six animals were adequate for each group.

\section{RESULTS}

Animal study. Twenty-nine rats were alive at the end of the study; one from PBS group died of limb necrosis. No significant differences were found for survival among the five groups $(P=0.38)$.

Sham-operated rats were not subjected to thrombosis procedure. Femoral artery was in normal pink color. In other groups, the 30min application of $\mathrm{FeCl} 3$ injury caused an immediate discoloration at the contact point with the femoral artery (Figure 2A-B). The rats were kept in the same animal room in optimum conditions. At follow up, it was noted that the rats in the sham group showed no skin discoloration at the dissection area or limping. Bluish-gray skin discoloration and limping were gradually increased in PBS group. A moderate skin discoloration and limping were observed in hBM-MSC and DFT groups. There were no symptoms or only mild symptoms in the rats from hBM-MSC + DFT group. All the rats were sacrificed 7 days after the procedure.

Thrombosis did not occur in sham operated

FIGURE 2. FeCl3 injury site and developing thrombus. (A) FeCl3 application to the left femoral artery. (B) Discolored site of $\mathrm{FeCl3}$ injury 30-min after injury. (C) Occlusive thrombus with evident thrombi and decreased vessel diameter in PBS group (HEE, 20x).

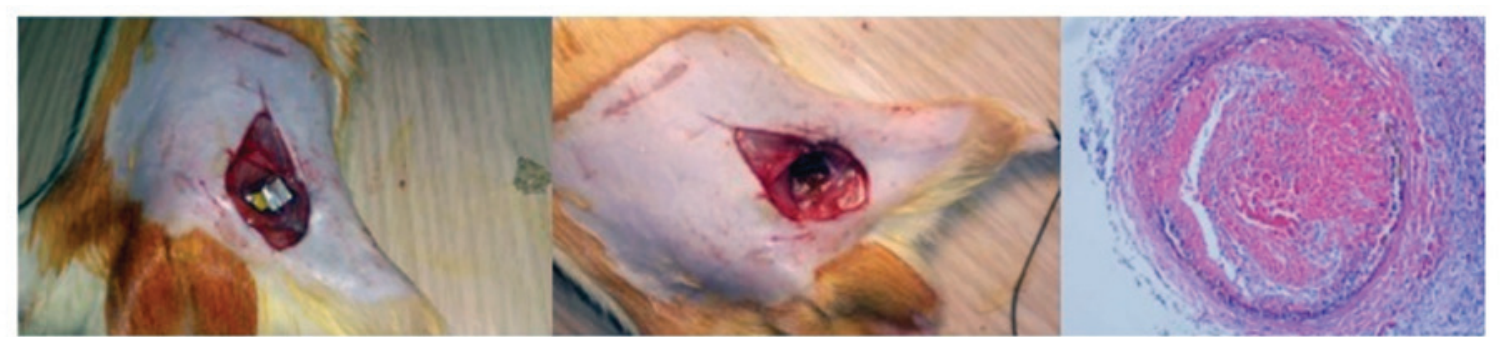

A)

B)
C) 
rats. In the PBS group, occlusive thrombus with evident thrombi and decreased vessel diameter were seen. There was no recanalization or neovascularization with negative immunostaining pattern (Figure 2C).

In group 3, only hBM-MSC was given after the procedure. Partial resolution of thrombi, formation of new channels around the vessel wall and adjacent to lumen area with positive immunostaining pattern by all antibodies were noted (Figure 3A1-A3). Group 4 received DFT alone following the procedure. Histopathological investigation of the vessel walls showed partial resolution of thrombi. Formation of new canals around the vessel wall and adjacent to lumen area were detected. There were $\mathrm{VWF}^{+}$, VEGFR- $1^{+}$and $\mathrm{CD} 68^{+}$cells around the thrombus; immunostaining for CD34 and CD133 were negative (Figure 3B1-B2). The rats in group 5 received hBM-MSC and DFT simultaneously. In histopathological investigation, near-complete resolution of the thrombi and new vascular channel formation were shown (Figure 3C1). There was positive immunostaining pattern with all antibodies. Figure $3 \mathrm{C} 2$ shows $\mathrm{VWF}^{+}$cells around the vessel wall. Although the use of hBM-
MSC or DFT alone enabled partial resolution of the thrombus, combining them resulted in nearcomplete resolution.

Neovascularization was two-fold higher in hBM-MSC + DFT treated rats $(11.6 \pm$ 2.4 channels) as compared to the rats in hBM-MSC $(3.8 \pm 2.7$ channels $)$ and DFT groups $(5.5 \pm 1.87$ channels) $(P<0.0001$ and $P=0.002$, respectively). There was no difference between the hBM-MSC and DFT groups $(P=0.46)$ (Figure $4 A)$.

VWF and VEGFR-1 immunostaing revelaled and identical pattern in hBM-MSC and DFT groups; $\mathrm{VWF}^{+}$cell numbers per field (100x) was $1.3 \pm 1.0$ in HBM-MSC group and $0.5 \pm 0.8$ in DFT group $(P=0.73)$ (Figure $4 B)$. VEGFR-1 ${ }^{+}$cells number per field (40x) was $9.5 \pm 10.2$ in hBMMSC group and $3.8 \pm 4.2$ in DFT group $(P=0.39)$. However, in hBM-MSC + DFT group, $\mathrm{VWF}^{+}$ $(9.1 \pm 3.0)$ and VEGFR- $1^{+}(25.5 \pm 6.2)$ cells number per field (40x) were significantly higher compared to hBM-MSC and DFT groups $(P \leq 0.005$ for all comparisons).

As expected, there was no $\mathrm{CD} 34^{+}$and $\mathrm{CD}_{133^{+}}$cells in the DFT group. Both antibodies showed a similar staining pattern in hBM-MSC treated groups $(P=0.91, P=0.42)$. Figure $4 C$ shows

FIGURE 3. (A1) Partial resolution of thrombi, formation of new channels around the vessel wall and adjacent to lumen area in hBM-MSC group (arrow) (HEE; 20x). (A2) CD68 ${ }^{+}$cells around the thrombi (arrow shows CD68 ${ }^{+}$cells) (40x).

(A3) VEGFR-1 $1^{+}$staining, new vessel formation around the thrombi (arrow shows VEGFR-1 ${ }^{+}$cells) (60x). (B1) Partial resolution of thrombi (star), intimal slimming, formation of new channels around the vessel wall and adjacent to lumen area in DFT group (arrow) (HEE; 20x). (B2) VEGFR-1+ staining, new vessel formation around the thrombi (arrows show VEGFR-1+ cells) (40x). (C1) In hBM-MSC + DFT group, near-complete resolution of the thrombi (left arrow) and new capillary formation (right arrow) (HEE; 20x). (C2) VWF' cells around the vessel wall (arrows show VWF cells) (40x)

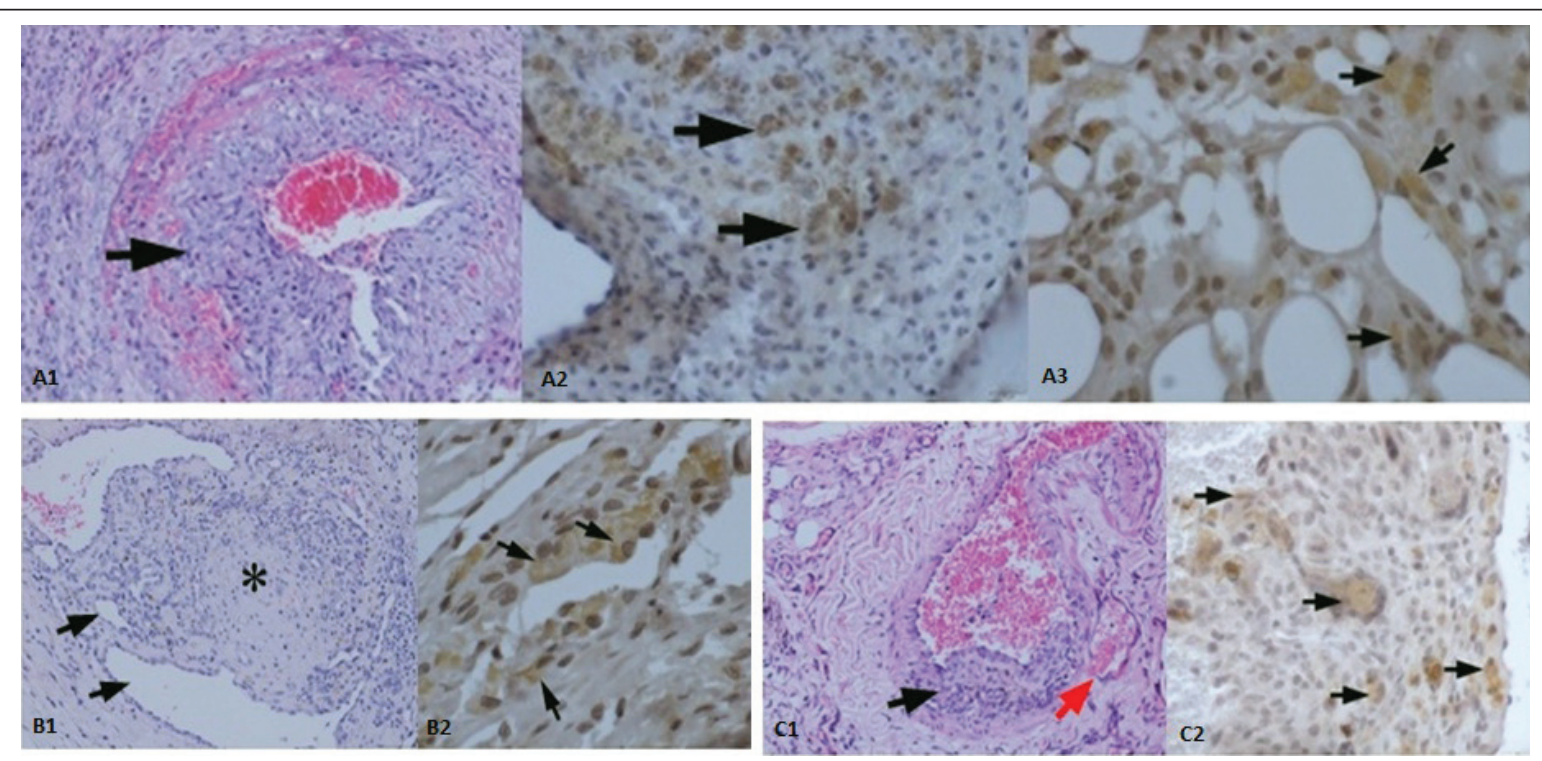


CD34 ${ }^{+}$staining in hBM-MSC treated groups. There was also no difference in terms of CD68 ${ }^{+}$ cell number per field (100x) between hBM-MSC $(28.8 \pm 1.8)$ and hBM-MSC + DFT $(33.6 \pm 6.9)$ groups $(P=0.16)$. A small number of $\mathrm{CD}^{+} 8^{+}$cells were found in DFT group (1.8 \pm 0.83$)$, which was the lowest count compared to hBM-MSC treated groups $(P<0.001)$.

Iron-labeled hBM-MSC homing to injured vessel wall was similar in hBM-MSC treated groups; cell number per field (100x) was $39.8 \pm 8.8$ in hBM-MSC and $41.6 \pm 6.8$ in hBM-MSC + DFT group $(P=0.69)$.

hBM-MSC homing was the highest in injured vessel wall and the lowest in the heart tissue in group $3(P=0.001) ; \beta-2$ microglobulin ${ }^{+} \mathrm{hBM}-$ MSCs number per field (100x) was $39.8 \pm 8.8$ in vessel wall, $32.3 \pm 4.3$ in lung tissue, $4.8 \pm 2.0$ in liver tissue, and $1.5 \pm 1.9$ in heart tissue $(P=0.001)$ (Figure 4D).

\section{DISCUSSION}

The organization and recanalization of thrombi are dynamic and complex processes that are influenced by microenvironmental factors, including cytokines, fibroblasts, macrophages, endothelial cells and leukocytes [4,25]. In recent years, endothelial progenitor cells (EPCs) have been found to play an important role in thrombotic process. Xiao-qiang et al., ${ }^{26}$ investigated the effects of transplanted bone marrow-derived EPCs on the vein microenvironment in a rat model of chronic vein thrombosis. They observed that endothelial cells surface markers, such as CD34, VEGF-2, and VWF were detectable, and cells grew gradually until they began to differentiate into mature endothelial cells. In this rat model of thrombosis, we found that immunostaining patterns for VWF and VEGFR-1 were similar in hBM-MSC and DFT groups, but significantly stronger in hBM-MSC + DFT group. Enhanced

FIGURE 4. (A) Neovascularization (channels per field, 100x) at day 7 after the procedure in rats treated groups. (B) VWF ${ }^{+}$cells number (per field, 100x). (C) CD34+ cells number (per field, 100x) in MSC treated groups. (D) Bar graph showing the highest hBM-MSC homing to injured vessel wall and the lowest to heart tissue ( $\beta-2$ microglobulin ${ }^{+}$cells number per field, 100x)
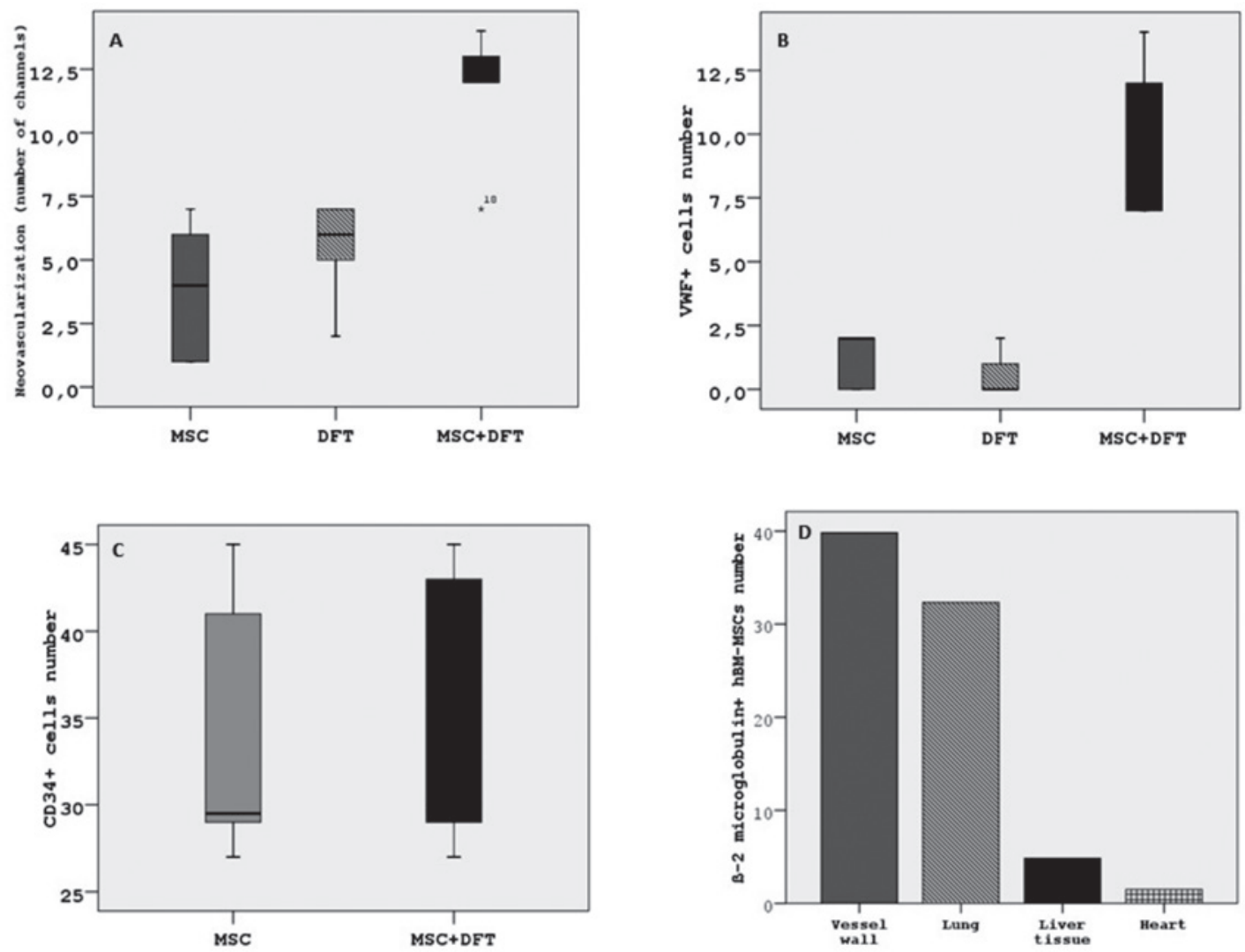

MSC: Mesenchymal stem cells.

DFT: Defibrotide. 
new capillary formation was observed when MSC and DFT were used combined.

Recently, Guerin et al., ${ }^{9}$ isolated embryoniclike stem cells from the patients with critical limb ischemia and studied their potential to differentiate into vascular lineages in a mouse model. The authors reported that stem cells were a potential new source of therapeutic cells that might give rise to cells of the endothelial lineage in humans. ${ }^{9}$

DFT can modulate endothelial cell function and have an antithrombotic effect on arteries, veins and capillaries. ${ }^{14}$ It was showed that DFT directly interferes with migration and tube formation of endothelial cells. ${ }^{16}$ Different properties of DFT [pro-fibrinolytic, antithrombotic-thrombolytic, anti-ischemic (heart, liver, kidney), anti-shock and anti-atherosclerotic] have confirmed its anti-endothelial cell activation activity. ${ }^{15}$ It has been found that DFT was useful especially in ischemic and microthrombotic conditions and vascular disorders. ${ }^{27,28}$

Here, we used hBM-MSC alone and together with DFT to enable new vascular formation in thrombosed vessels. Although the use of hBMMSC or DFT alone enabled partial resolution of the thrombus, combining them resulted in nearcomplete resolution. We also showed that hBMMSC homing number was the highest in injured vessel wall and the lowest in the heart tissue. Our observations suggest that transplantation of MSCs can change the thrombotic microenvironment and provide a molecular mechanism for resolution and neovascularization of the thrombi.

As a limitation of the study, although we aimed to conduct a trial on the treatment of femoral arterial thrombosis in newborns, experiments were carried out on male adult Sprague-Dawley rats because of the small calibers of the femoral arteries in newborn rats. Thus this may mask the application of the results in pediatric population. However, this study may give some inspiration for further research.

\section{CONCLUSION}

To our knowledge, this is the first to attempt to combine MSC and DFT to enhance thrombus resolution by promoting neovascularization. Neovascularization significantly increased by using combined treatment with DFT and hBM-MSC, but the molecular and cellular mechanisms that regulate this process still need to be clarified.

\section{REFERENCES}

1. Kuhle S, Male C, Mitchell L. Developmental hemostasis: pro- and anticoagulant systems during childhood. Semin Thromb Hemost 2003;29(4):329-38.

2. Brotschi B, Hug MI, Latal B, Neuhaus D, et al. Incidence and predictors of indwelling arterial catheter-related thrombosis in children. J Thromb Haemost 2011;9(6):1157-62.

3. Dumond AA, da Cruz E, Almodovar MC, Friesen RH. Femoral artery catheterization in neonates and infants. Pediatr Crit Care Med 2012;13(1):39-41.

4. McGuinness CL, Humphries J, Waltham M, Burnand KG, et al. Recruitment of labelled monocytes by experimental venous thrombi. Thromb Haemost 2001;85(6):1018-24.

5. Waltham M, Burnand KG, Collins M, Smith A. Vascular endothelial growth factor and basic fibroblast growth factor are found in resolving venous thrombi. J Vasc Surg 2000;32(5):988-96.

6. Waltham M, Burnand K, Fenske C, Modarai B, et al. Vascular endothelial growth factor naked DNA gene transfer enhances thrombus recanalization and resolution. J Vasc Surg 2005;42(6):1183-9.

7. Caplan AI. Adult mesenchymal stem cells for tissue engineering versus regenerative medicine. J Cell Physiol 2007;213(2):341-7.

8. Shake JG, Gruber PJ, Baumgartner WA, Senechal G, et al. Mesenchymal stem cell implantation in a swine myocardial infarct model: Engraftment and functional effects. Ann Thorac Surg 2002;73(6):1919-26.

9. Guerin CL, Loyer X, Vilar J, Cras A, et al. Bone-marrowderived very small embryonic-like stem cells in patients with critical leg ischaemia: evidence of vasculogenic potential. Thromb Haemost 2015;113(5):1084-94.

10. Gnecchi M, Melo LG. Bone marrow-derived mesenchymal stem cells: isolation, expansion, characterization, viral transduction, and production of conditioned medium. Methods Mol Biol 2009;482:281-94.

11. Haynesworth SE, Baber MA, Caplan AI. Cytokine expression by human marrow-derived mesenchymal progenitor cells in vitro: effects of dexamethasone and IL-1 alpha. J Cell Physiol 1996;166(3):585-92.

12. Pillekamp F, Reppel M, Brockmeier K, Hescheler J. Stem cells and their potential relevance to pediatric cardiology. Cardiol Young 2006;16(2):117-24.

13. Tayman C, Uckan D, Kilic E, Ulus AT, et al. Mesenchymal stem cell therapy in necrotizing enterocolitis: a rat study. Pediatr Res 2011;70(5):489-94.

14. Niada R, Mantovani M, Prino G, Pescador R, et al. PGI2generation and antithrombotic activity of orally administered DFT. Pharmacol Res Comm 1982;14(10):949-57.

15. Pescador R, Capuzzi L, Mantovani M, Fulgenzi A, et al. Defibrotide: properties and clinical use of an old/new drug. Vascul Pharmacol 2013;59(1-2):1-10.

16. Koehl GE, Geissler EK, Iacobelli M, FreiC, et al. Defibrotide: an endothelium protecting and stabilizing drug, has an anti-angiogenic potential in vitro and in vivo. Cancer Biol Ther 2007;6(5):686-90.

17. Fontana L, Partridge L, Longo VD. Extending healthy life span--from yeast to humans. Science 2010;328(5976):321-6.

18. Grodzinska L, Konig E, Schror K. DFT is equipotent to urokinase in stimulating arterial and venous thrombolysis. Pharmacol Res Comm 1987;19:609-15.

19. Paul W, Gresele P, Momi S, Bianchi G, et al. The effect of defibrotide on thromboembolism in the pulmonary vasculature of mice and rabbits and in the cerebral vasculature of rabbits. Br J Pharmacol 1993;110(4):1565-71.

20. Tettamanti R, Bianchi, G, Vitte PA, Kato G, et al. A novel insight into the mechanism of the antithrombotic action of DFT. LIFE Sci 1992;51(19):1545-55. 
21. SurinWR, PrakashP, BarthwalMK, DikshitM.Optimization of ferric chloride induced thrombosis model in rats: effect of anti-platelet and anti-coagulant drugs. J Pharmacol Toxicol Methods 2010;61(3):287-91.

22. Uckan D, Kilic E, Sharafi P, Kazik M, et al. Adipocyte differentiation defect in mesenchymal stromal cells of patients with malignant infantile osteopetrosis. Cytotherapy 2009;11(4):392-402.

23. Dominici M, LeBlancK, Mueller I, Slapper-Cortenbach I, et al. Minimal criteria for defining multipotent mesenchymal stromal cells. The International Society for Cellular Therapy position statement. Cytotherapy 2006;8(4):315-7.

24. Amsalem Y, Mardor Y, Feinberg MS, Landa N, et al. Ironoxide labeling and outcome of transplanted mesenchymal stem cells in the infarcted myocardium. Circulation 2007;116(11 Suppl):I38-45.

25. Modarai B, Burnand KG, Humphries J, Waltham M, et al. The role of neovascularisation in the resolution of venous thrombus. Thromb Haemost 2005;93(5):801-9.

26. Li XQ, Meng QY, Wu HR. Effects of bone marrow-derived endothelial progenitor cell transplantation on vein microenvironment in a rat model of chronic thrombosis. Chin Med J (Engl) 2007;120(24):2245-9.

27. Coccheri S, Biagi G. Defibrotide. Cardiovasc Drug Rev 1991;9(2):172-96.

28. Palmer KJ, Goa KL. Defibrotide. A review of its pharmacodynamic and pharmacokinetic properties, and therapeutic use in vascular disorders. Drugs 1993;45(2): 259-94. 